\title{
Patient lost in the private healthcare mix - Broomberg
}

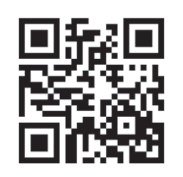

The South African (SA) healthcare system is organised around the needs of just about every stakeholder except the patient, with a highly fragmented, 'siloed' approach where integrated care is the exception and not the rule, says Discovery Health CEO Jonny Broomberg.

Speaking at a healthcare summit held at Discovery's headquarters in Sandton, Johannesburg, on 28 May, Broomberg said he was excited by the Competitions Commission inquiry into the private healthcare sector because it would bring to the fore much-needed information around real cost drivers. Using US-generated data (because their healthcare system is similar to SA's), he said that serious empirical research has shown that wastage in their system ranged between $21 \%$ and $47 \%$. He conservatively put SA's wastage at $21 \%$. 'This doesn't generate clinical value for patients. We have the undesirable fee-for-service and separation of health professionals and hospitals. If you tally up over-treatment (5.9\%), administrative complexity (4\%), failures of care delivery (3.8\%), pricing

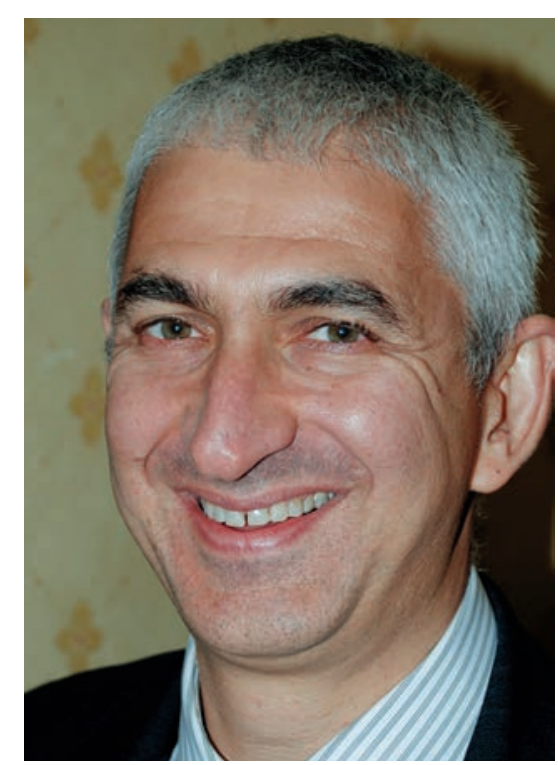

the highly respected New England Journal of Medicine.

Volumes push medical inflation beyond the consumer price index (CPI)

Singling out SA's scarce human skills as a major driver of medical inflation, Broomberg said that in the manufacturing sector, amazing new technologies usually brought down costs. However, in the healthcare sector, 'no matter what you do, you somehow can't bring the price down - you still have to have the surgeon to do the work' While the inflation debate had historically focused on prices that hospitals and doctors charged, Discovery's data over the past 5 years showed that on average premium inflation had increased by $11.5 \%$ - with $7 \%$ related to prices and $4.5 \%$ linked to volume. This meant that in reality, price increases actually tracked 'incredibly close' to the CPI - but with the average medical scheme member consuming $4.5 \%$ more doctor and hospital visits than the year before (Fig. 1). Broomberg said that last year Discovery members made 6.6 million GP visits and 7.9 
million specialist visits (totalling R16 billion, or $48 \%$ of expenditure), and that there were 592000 hospital admissions with 1.9 million days in hospital (costing R12.4 billion, or $37 \%$ of expenditure), while 486000 lives were registered for chronic medication (costing R5.1 billion, or 15\% of expenditure) (Fig. 2).

Families belonging to Discovery's top medical aid plan (Classic Comprehensive) and spending more than R500 000 per annum totalled 15/10 000 in the year 2000 . By last year this had increased five-fold (to 79/10 000) - a 'crisp illustration' of volume pressures. Premiums meanwhile had increased by between one and a half times and double. The 'tidal wave' of noncommunicable diseases pushed up the disease burden over the past 5 years by $17 \%$, but with two-thirds of Discovery's members now on their Vitality health and fitness programme, 'we think our numbers are going up more slowly? Broomberg said chronic patients claimed four times as much as the average member. The disease burden and the unintended consequences of legislation aggravated inefficiencies and costs, and his company's agenda was now based on the most innovative and progressive healthcare systems in the world. 'It's really about value equals quality over cost, putting the patient at the centre. Our [the SA] system is organised around the needs of just about every stakeholder other than the patient $\ldots$ doctors, hospitals, funders ... we haven't designed a system to say what fits the consumer.'

\section{Create multidisciplinary teams to save lives and money}

A move towards multidisciplinary teams looking after complicated cases would dramatically reduce costs and improve outcomes. He singled out Dr Carol Benn, who has established three thriving breast cancer clinics at the Chris Hani Baragwanath, Helen Joseph and Milpark hospitals, which draw on the best available expertise in every discipline associated with breast cancer. Daily conferences discuss cases, with input from every professional adding to the overall patient care. Besides fragmentation, the paucity of measurement in healthcare outcomes and cost ('if you can't measure it, you can't manage it') was a constant thorn in the side of value-based delivery. "We need to bring measurement into the way we reward and pay. Linked to that is a shift away from fee for service to what the literature globally calls "bundled payments" (e.g. capitation). We need to move towards value and quality, not volume, he added.

\section{Do the basics right - or lose lives}

Using the analogy of standard preflight checks in the aviation industry, Broomberg said that medicine had a lot to learn from this sector. He showed slides of dramatic variations in the death rates for acute heart attacks at individual SA private hospitals (averaging about $7.4 \%$, the global average). However, in many emergency rooms the essential standard protocols of administering aspirin and beta-blockers the moment the patient arrived were often not followed, accounting for too many unnecessary deaths. The same variation from hospital to hospital existed for patients on renal dialysis, with complications setting in and infected patients returning to hospital, where they often died. By introducing a standardised kidney care programme, Discovery had seen a $5 \%$ improvement in dialysis scores

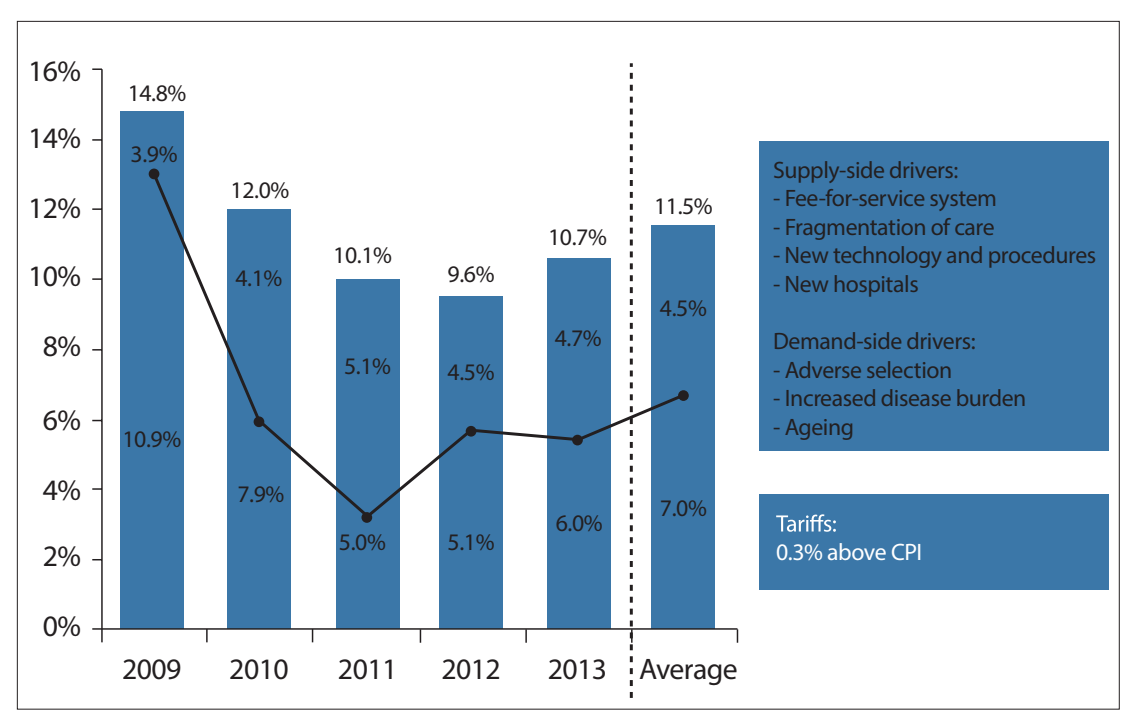

Fig. 1. SA medical inflation is not all about tariffs - volume of services is a critical cost driver. (Source: Discovery Health.)

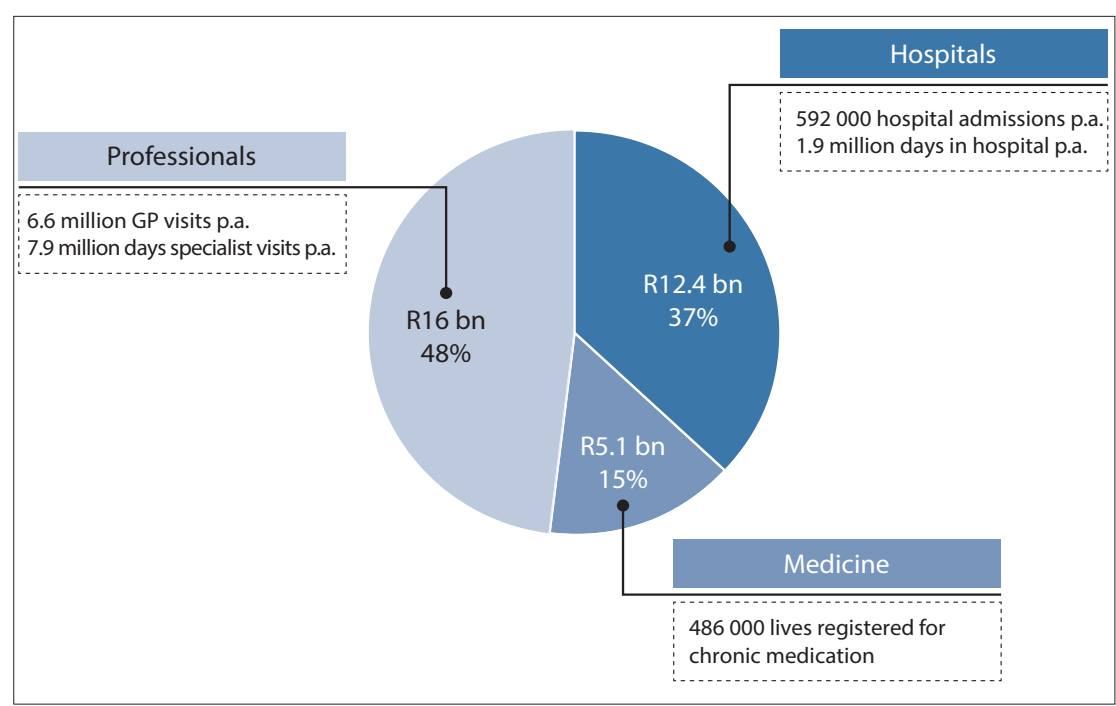

Fig. 2. Breakdown of Discovery Health claims expenditure, 2013. (Source: Discovery Health.)

across their patient population with an exponential increase in lives saved. The setup involved kidney specialists and dialysis centres submitting data to Discovery on the key elements of dialysis, with built-in feedback. Another lifesaving intervention was to cut down dramatically on central line infections. Patients in intensive care units often had a catheter in the chest rather than a drip in the arm. By ensuring that doctors scrubbed hands, put on masks and gowns and observed basic hygiene precautions (which he said took 'years of work with hospital groups, and then doctors'), Discovery had brought down central line infections among their members (and obviously other patients) from 4/1 000 hospital days to just 1/1000 hospital days. 'Getting such an infection doubles hospital costs and has a $12-25 \%$ mortality rate, Broomberg added. Data on antibiotic 
use showed a five-fold variation between hospitals. Adjusted for severity of condition, the data revealed that $77 \%$ of inpatients were on antibiotics, with 'huge overuse' and wide geographical variations. 'Healthcare policy and quality experts will always tell you that where you find variation in a healthcare system, you've got problems,' he added. His company was 'just scraping the tip of the iceberg', with interventions that turned quality measurements into better outcomes - and would later this year begin paying doctors more for improved results. 'You can also ask really simple questions, like do you have a protocol for when a patient arrives with a heart attack?', he added.

\section{Cash-plan members in the cross-hairs}

Broomberg's presentation revealed that Discovery keeps an eagle eye on members who also have cash plans. This cohort of patients has hospital admission rates 2.5 5.5 times higher than those who don't with doctor syndicates being exposed. 'Take a cash-strapped family that has a hospital cash plan that pays R5 000 per day after day 3 of admission. The temptation to get the doctor to keep you in for 4 or 5 days is huge - and Discovery ends up with a R50 000 hospital bill!' Most medical schemes were not being sufficiently proactive, with 'big scams' involving the active collaboration of crooked doctors constantly on the go. The biggest culprit geographically was KwaZuluNatal. Asked to specify conditions diagnosed to 'justify' medically unsound hospital stays, Broomberg cited haematemesis (vomiting blood, usually involving the upper gastrointestinal tract) as a top culprit. $\mathrm{He}$ said that in general, fraud cases had only been proven against a relatively small number of doctors. Where clear identification was possible, Discovery terminated the patient's membership, reported the doctor to the Health Professions Council of South Africa and cancelled any contract they had with the doctor before recovering the money. "What we're trying to do is shut it off at source. If you have a known cash plan we check up more carefully. I think we've created a halo effect with some people moving to other medical schemes.' Discovery had clawed back R288 million in fraud last year (representing about $1 \%$ of contributions). Broomberg estimates the total national healthcare fraud bill at about R8.22 billion. 'The more bodies we throw at it, the more we get back. Last year we got $14 \%$ more back than the previous year - and we haven't yet reached the bottom of the pond.' Other scams included pharmacies selling 'nappies and cosmetics' and submitting them as medical claims, and card-sharing. The 'halo effect' extended to GPs bust for fraud. 'You get behaviour change in addition to getting your money back on the day'. He appealed for other medical schemes to work with his company: 'We all have to squeeze down, or it's just a water bed - you squeeze down here and it pops up in another scheme.' Discovery did not deny that cash plans had a role, 'but you don't want them abused and driving up health costs?

\section{'Naming and shaming' hospitals counterproductive}

Broomberg said that sustained diplomacy was required to get hospitals, doctors and pathology laboratories to work with medical aids in providing data, 'otherwise you can push them underground and voluntary reporting becomes impossible'. Asked if he was prepared to release a list of 'which hospitals to avoid', Broomberg said his company tried this 5 years ago, to howls of protest over contested data and a quick exit from the public domain. Some of these clinical coding data were a bit inaccurate, he confessed, adding that the problem was being speedily rectified to increase the data robustness. This included discerning where weaknesses were attributable to the hospital or the doctors - or both. 'It's one thing to go to them privately and give feedback, it's quite another if you go public - if you're wrong there can be a lot of damage.'

\section{Chris Bateman}

chrisb@hmpg.co.za

S Afr Med J 2014;104(8):533-535.

DOI:10.7196/SAMJ.8625 